\title{
Syringomatous carcinoma of the eyelid and orbit: a clinical and histopathological challenge
}

\author{
V P T Hoppenreijs, T T Q Reuser, C M Mooy, R J W de Keizer, M Ph Mourits
}

\begin{abstract}
Aims-To present three patients with a syringomatous carcinoma (SC). SC is a rare cutaneous neoplasm, most frequently situated on the face and scalp and histologically characterised by an infiltrative pattern of basaloid or squamous cells, a desmoplastic stromal reaction, keratin filled cysts, and granular structures.

Methods-The clinical histories of the patients with a SC were investigated retrospectively.

Results-Patient 1 had a benign appearing tumour of the lower eyelid. Five tumour excisions were necessary to remove the SC completely. Patient 2 had a tumour on the lateral part of the lower eyelid and in the medial canthal area. The histopathological findings revealed a squamous cell carcinoma, later revised as a SC. In spite of two excisions and one microscopically controlled excision, a recurrence occurred. An exenteration orbitae was recommended. Patient 3, known to have a history of multiple malignant skin tumours after kidney transplantation and use of cyclosporin, presented with a firm mass in the eyebrow region and in the nasal area of the orbit. The pathological diagnosis of this adnexal tumour was difficult. An exenteration was recommended.
\end{abstract}

Conclusions-SC is a benign appearing but extremely invasive, locally destructive, slowly growing adnexal tumour, derived from eccrine sweat glands. It is often mistaken, both clinically and microscopically, for other benign and malignant entities. The tumour recurrence is high due to extensive perineural invasion, but regional or distant metastases are rare. The local aggressive nature of the tumour and the high recurrence rate may necessitate mutilating procedures. Optimal treatment consists of a complete microscopically controlled surgical excision with clear surgical margins.

(Br f Ophthalmol 1997;81:668-672)

Syringomatous carcinoma is a rare malignant skin appendageal tumour derived from sweat glands, first described by Goldstein et al in 1982 as a microcystic adnexal carcinoma (see Table 1). ${ }^{1-2}$ Since it was recognised there has been much controversy about its nomenclature. Since 1970, a variety of synonyms have been given to some carcinomas with eccrine differentiation, among them syringoid eccrine carcinomas, microcystic adnexal carcinoma, malignant syringoma, sclerosing sweat duct (syringomatous) carcinoma, and sweat gland carcinoma with syringomatous features. ${ }^{3-10} \mathrm{It}$ is currently believed that these are different names for the same neoplastic process with different degrees of differentiation. The entire group is termed 'syringomatous carcinoma', characterised by cutaneous involvement, invasive destructive local growth, skeletal muscle, and perineural invasion. ${ }^{1}$

We describe three patients with a syringomatous carcinoma of the eyelid and in the periorbital region, demonstrating the local aggressive nature of this tumour. The purpose of this study was to describe the clinical features, the histopathological findings, the treatment, and the difficulties encountered in diagnosing this tumour, both clinically and histopathologically, in our patients.

\section{Case reports}

CASE 1

A 50-year-old woman presented at the Academic Hospital Utrecht with an irritating and itching cutaneous nodule on the medial part of the right lower eyelid. The nodule measured 5 $\times 4 \mathrm{~mm}$, was painless, benign appearing, firm, and involved the lid margin just near the lacrimal punctum. For cosmetic reasons, the patient underwent surgical excision of the benign appearing tumour under local anaesthesia. However, histopathological examination revealed a moderately differentiated syringomatous carcinoma which was not completely removed. One month later, a wide full thickness excision of the lower eyelid was performed. The specimen measured $9 \times 6 \mathrm{~mm}$ and only the medial part of this specimen appeared to be free of tumour. During the next month, two more excisions were performed and again the lateral margins of the specimen were not free of tumour. Two weeks after the last surgery, a full thickness excision of the complete lower eyelid and excision of some periorbital tissue were necessary to remove the tumour completely. One month after the complete re-excision, the lower eyelid was reconstructed. The histopathological findings showed a syringomatous pattern (Fig 1), and neoplastic epithelial cells arranged in interconnecting cords with microcystic areas. Nests, cords, and tubules of the tumour extended into the dermis and into the orbicularis muscle (Fig 2,3 , and 4). There was extensive perineural infiltration by neoplastic cells (Figs 5). Sclerosis of stroma around the cords was present. Tumour cells were not connected to the epidermis. The immunohistochemical analysis
Accepted for publication 10 April 1997 


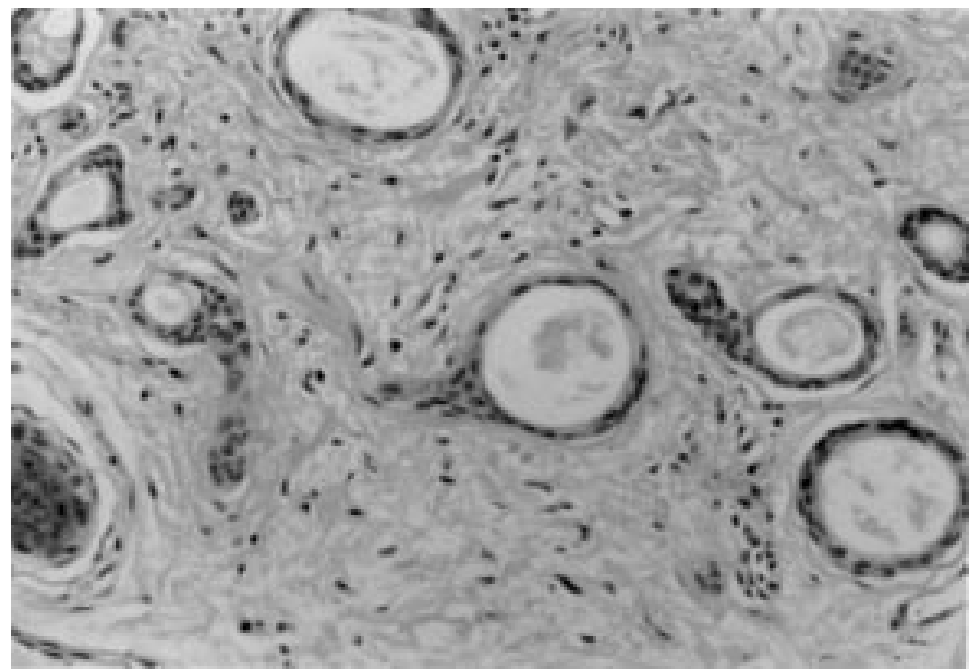

Figure 1 Syringoma (a benign sweat gland tumour), showing the characteristic syringomatous pattern. Numerous small comma-shaped ducts are embedded in fibrous stroma. Amorphous eosinophilic debris is often within the ductal lumina. Anaplasia and invasion are not observed in benign sweat gland tumour. The benign tumour does not show perineural, subcutaneous or muscle infiltration (in contrast with the syringomatous carcinoma). Magnification $\times 192$.

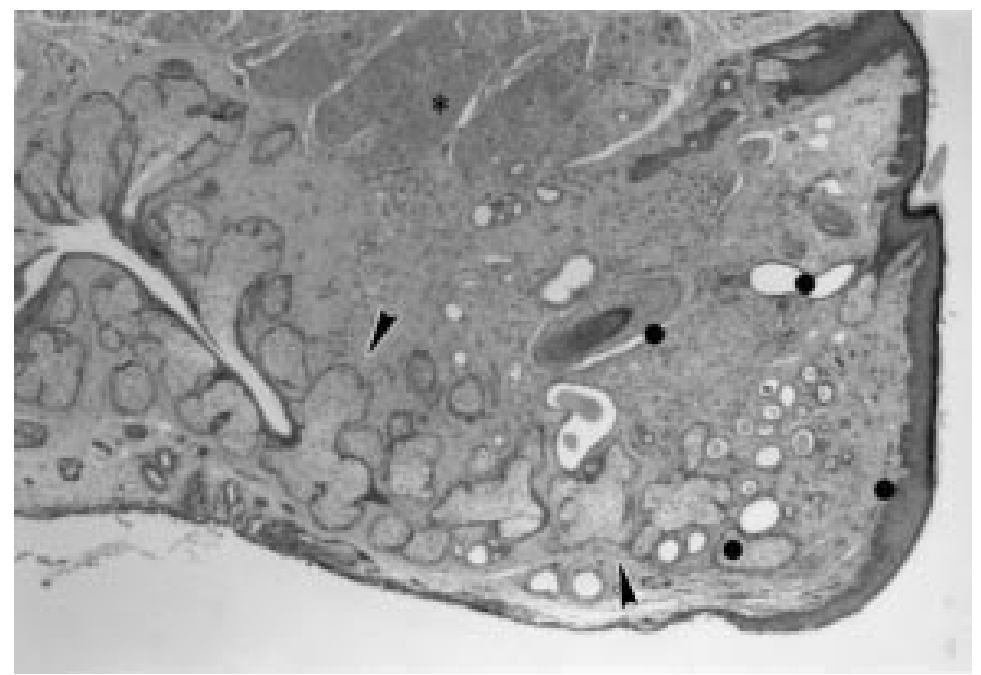

Figure 2 Survey light micrograph of a specimen of the lower eyelid of patient 2, demonstrating a syringomatous carcinoma infiltrating in muscle (asterisk) and between sebaceous structures (arrowheads). The area between the circles is magnified in Figure 3. Magnification $\times 30$. excisional biopsy from the first lesion revealed a squamous cell carcinoma and from the second lesion a Bowen's disease. The lesions were treated by ionising radiation. Eight months later, the patient was referred to the department of ophthalmology of the Academic Hospital Nijmegen with a firm, plaque-like lesion with poorly circumscribed margins, telangiectasias, and central ulceration at the lower eyelid. After an incisional biopsy of the lateral canthus and an excisional biopsy of the medial part of the eyelid, the lower eyelid was reconstructed. Pathological examination of the incisional biopsy showed a squamous cell carcinoma while the excisional biopsy showed a moderately differentiated squamous cell carcinoma in the superficial layers merging into a syringomatous carcinoma in the deep layers. The resected specimen, however, did not confirm complete excision.

Four months later, microscopically controlled surgical excision was performed utilising frozen section control. Most of the lower eyelid, the lateral canthus, and part of the upper eyelid were excised. The pathological findings included nests and cysts of cytologically uniform, squamoid cells that were randomly dispersed in a dense oedematous collagenous stroma. In some specimens, branching of tubular structures and aggregations of neoplastic cells gave comma-like shapes. The microcysts contain lamellated keratinaceous material resembling hair matrix, and some may demonstrate extensive squamous metaplasia. Perineural infiltration of tumour cells was present. The immunohistochemical analysis was consistent with syringomatous carcinoma (see case 1). Although the superficial part of the tumour showed some aspects of a squamous cell carcinoma, the overall picture of the specimens disclosed a syringomatous carcinoma. This diagnosis was confirmed by re-evaluating previous specimens. Unfortunately, the tumour extended to the margins of resection. Four months later, the specimen of an incisional biopsy revealed a recurrence of the tumour. The patient's best corrected visual acuity was 0.4 in both pseudophakic eyes. Although orbital exenteration with microscopic analysis of the margins was advised, the patient declined any further surgery. Radiotherapy of the involved area was performed. membrane antiy trup, Denmark), anti-CEA (carcinoembryonic antigen, dilution 1:100, Bio Genex, San Ramon USA), AE1/AE3 (cytokeratins, dilution 1:200 in an overnight incubation protocol at $4^{\circ} \mathrm{C}$, Bio Genex, San Ramon, USA), and $S 100$ protein (expressed by eccrine sweat gland cells, dilution 1:5000, Glostrup, Denmark) (see Discussion). These histological and immunohistochemical analyses were consistent with the diagnosis of syringomatous eccrine carcinoma.

CASE 2

A 83-year-old white woman presented with a slow growing, crusty, painless, plaque-like lesion at the lateral $3 / 4$ side of the right lower eyelid and a second isolated erythematous crusty nodule in the medial lower eyelid and medial canthal region of the same side. An

\section{CASE 3}

A 54-year-old man had an elaborate clinical history including two kidney transplants in 1970 and 1990 with cyclosporin treatment to prevent rejection. Between 1983 and 1995, the patient developed several benign lesions (naevus naevocellularis, papillomas), premalignant lesions (actinic keratosis), and multiple malignant tumours of the skin, such as squamous cell carcinomas (right eyelid, temple, back of the hand, neck, preauricular skin, back, wrists, nostril, forehead, right medial canthal area, and many recurrences) and basal cell carcinomas (upper arm, nose, neck, temple, under arm, nostril, and recurrences) (Fig 6). Initial biopsy of the tumour present in the medial canthal 


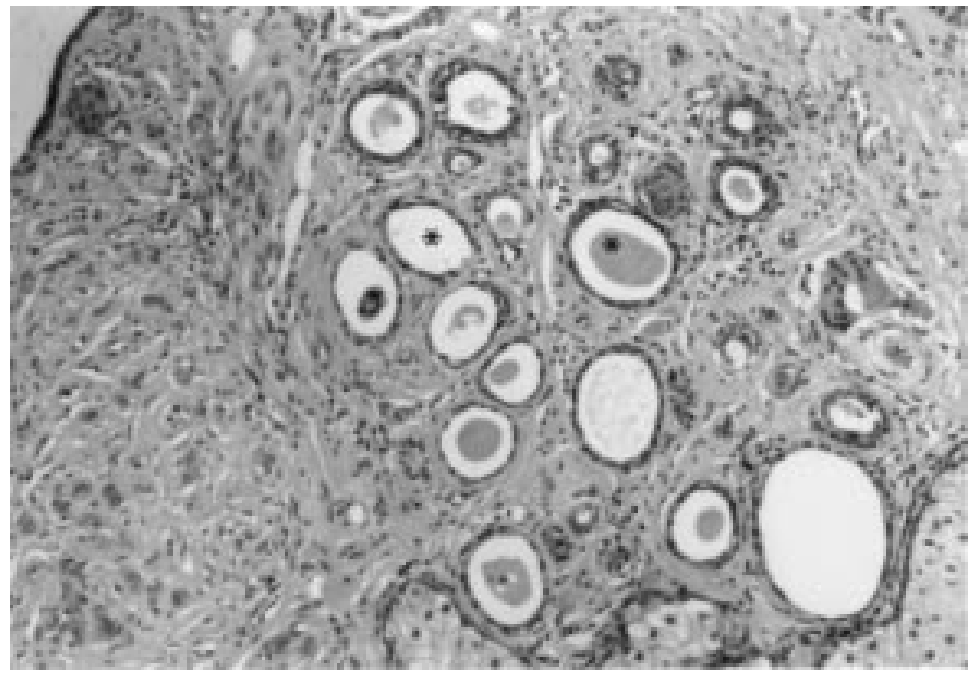

Figure 3 Detail of the syringomatous carcinoma (see Fig 2) with tubules simulating syringoma and less differentiated cords. Magnification $\times 120$.

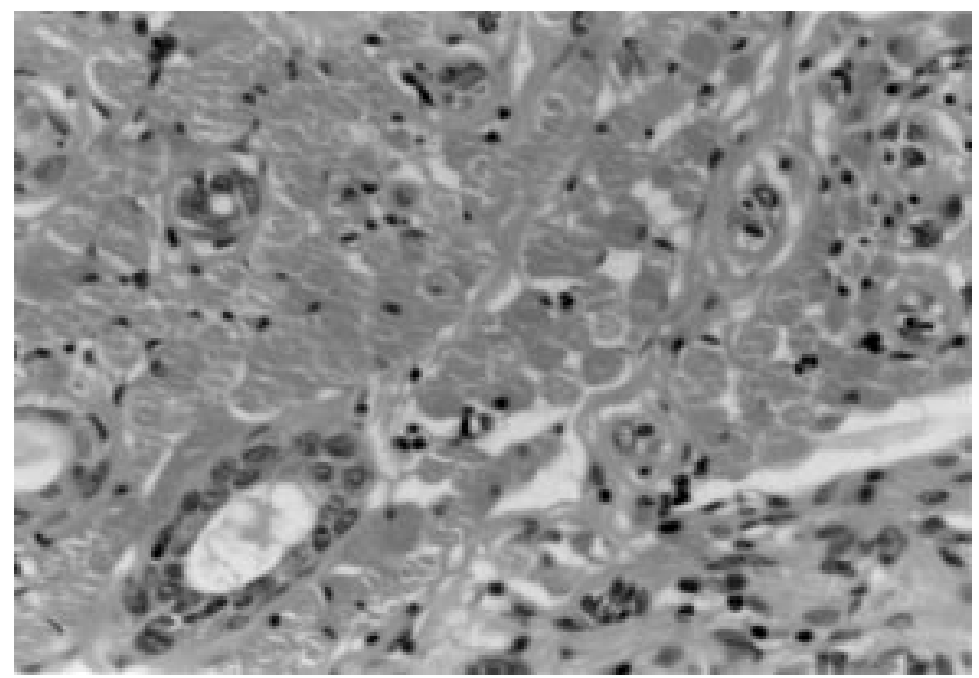

Figure 4 Syringomatous carcinoma: detail of muscle infiltration. Magnification $\times 300$.

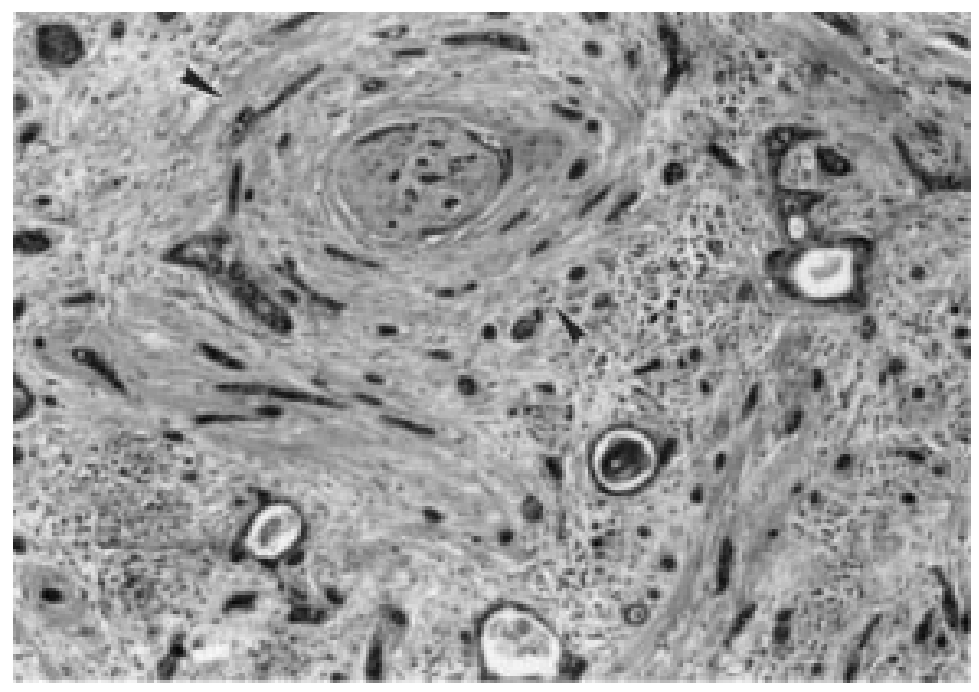

Figure 5 Syringomatous carcinoma: detail of perineural invasion (arrowheads). Magnification $\times 300$. and eyebrow region was interpreted as an eccrine cell carcinoma. In September 1995, the patient was referred to the Academic Hospital Leiden with a proptosis of $2 \mathrm{~mm}$, progressive chemosis, and motility disturbances (adduction, abduction) of the right eye. The best corrected visual acuity was 0.9 in the right eye and $0.5 / 60$ in the left eye. A firm mass was palpable in the nasal area. A magnetic resonance image scan showed that the tumour invaded the medial aspect of the right orbit near the trochlea, with invasion into the frontal bone. The biopsy specimen was re-examined and again a syringoid eccrine carcinoma was diagnosed, later revised as squamous cell carcinoma. After consultation with several orbital teams, an exenteration of the orbital contents was recommended. Because of the poor clinical condition of the patient, this operation was not possible. Radiation therapy was considered but the skin malignancies and the radiation induced impairment of vision restrain us from performing this therapy. It was decided to apply palliative therapy if necessary.

Difficulties in diagnosing were present. The specimens from the medial canthus area and forehead were reevaluated by other pathologists (Erasmus University, Rotterdam). The specimens revealed a squamoid tumour, without microcystic differentiation, and no connection of tumour to the epidermis. Within the tumour, there was local presence of hair, some mitotic figures, a marked perineural invasion, and absence of desmoplastic stroma. Some reactive atypical sweat glands were present. The tumour cells were strongly positive for anti-cytokeratin and anti-EMA, and negative for CEA and for S100. This picture was consistent with an adnexal carcinoma with pilar differentiation.

\section{Discussion}

The ocular adnexa include hair follicles, sebaceous glands (meibomian, Zeis, and caruncle glands), and sweat glands (apocrine and eccrine). The vast majority of the conditions are fortunately benign. However, some are malignant and may have aggressive features. A general categorisation of adnexal tumours is given in Table 1 .

Syringomatous carcinoma (SC) is derived from the sweat glands and is characterised by tubular features and eccrine differentiation, which can, according to Abenoza and Ackerman, be classified further as: (1) well differentiated SC (main synonym: microcystic adnexal carcinomas), (2) moderately differentiated SC, and (3) poorly differentiated SC. Many synonyms can be found in literature, ${ }^{1}$ but they probably represent the same neoplastic process with different degrees of differentiation. The well differentiated form is most frequently found than the other two forms and will therefore be discussed below.

CLINICAL FEATURES

The sex distribution is about equal. ${ }^{11}$ The patients present most frequently in the fourth and fifth decades of life (age range 20s to $80 \mathrm{~s}) .{ }^{12}{ }^{12}$ Many patients have been aware of the 


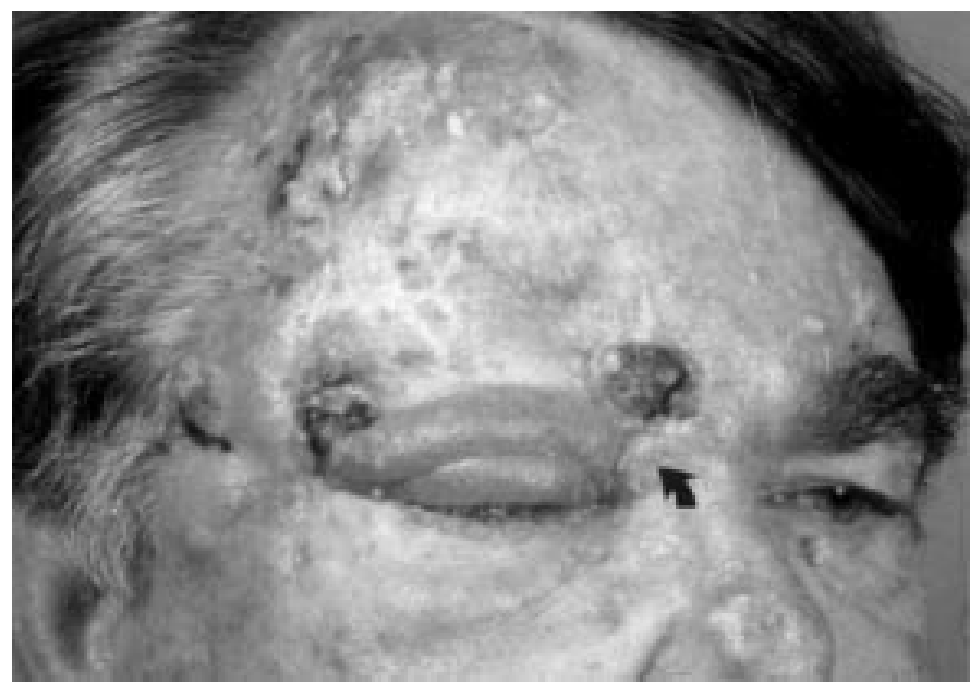

Figure 6 Patient 3 with multiple malignant tumours of the skin, such as squamous cell carcinomas (right eyelid, temple, neck, preauricular skin, nostril, forehead, right medial canthal area) and basal cell carcinomas (nose, neck, temple, nostril). A firm mass was palpable in the nasal area (arrow).

Table 1 Adnexal tumours (located on eyelids)

Sweat glands:

(a) Apocrine tumours:

Benign: hidrocystoma

syringocystadenoma papilliferum

hidradenoma papilliferum

adenoma

cylindroma

Malignant: adenocarcinoma (of the gland of Moll)

(b) Eccrine tumours

Benign: hidrocystoma

syringoma

chondroid syringoma (mixed

tumour)

acrospiroma (clear cell

hidradenoma)

spiradenoma

Malignant: malignant forms of 'benign eccrine tumours'

primary mucinous carcinoma

(adenocystic)

primary infiltrating signet ring

carcinoma

sclerosing sweat duct carcinoma or

syringomatous carcinoma

II Pilar tumours (hair follicles)

III Sebaceous tumours

IV Lesions simulating primary adnexal tumours

existence of the neoplasms for many years before they seek consultation. ${ }^{711}$ SC are mostly situated on the head and neck with a predilection for the centrofacial region (about $85 \%$ ) (mainly upper and lower lip). Less frequent sites include the eyebrow region, the nasolabial fold, nose, cheek, scalp, chin, and periorbital skin. Although the face is the most common site for well differentiated syringomatous carcinomas, the moderately differentiated ones are mostly situated on the scalp and, rarely, on the face. ${ }^{1}$ Until now, only six cases of 'microcystic adnexal carcinoma' (the well differentiated form of SC) were reported in the orbital area, involving the eyebrow region (three cases), ${ }^{13-15}$ the lower eyelid (two cases), ${ }^{10}$ and upper eyelid (one case). ${ }^{15}$ In our two cases, the neoplasm was located in the lower eyelid with periorbital invasion. One immunocompromised patient (case 3) showed eyebrow/ upper eyelid and secondary orbital involvement.
Clinically, lesions usually manifest as a slowly growing, flesh or pale/yellow coloured, solitary, firm, indurated nodule or plaque, often with diffuse, ill defined margins and overlying telangiectasia. The overlying epidermis may appear normal, atrophic, or scaly. Ulceration is unusual. The clinical differential diagnosis includes morphea-like basal cell carcinoma. Clinical and histopathological evaluation have to be performed for these imitations of eyelid tumours. ${ }^{16}$

\section{HISTOPATHOLOGICAL FINDINGS}

The microscopic appearance of SC mimics that of several benign sweat gland tumours, including benign syringoma (Fig 1). The main characteristics are syringomatous pattern (Fig 1) (including formation of tubules), formation of cysts containing cornefied cells, extensions of neoplastic cells into subcutaneous tissues, sclerotic stroma, and rare or absent mitosis. SC is basically composed of nests, cords (mostly interconnecting), tubules, and cysts of cytologically uniform, cuboidal, or flattened squamoid cells that are randomly dispersed in a dense collagenous stroma (desmoplasia) (Figs 2 and 3). Branching of tubular structures and aggregations of tumour cells that have the shapes vaguely resembling 'tadpoles' or 'commas' are seen. The histological diagnosis can be confirmed by immunohistochemical analysis: positivity for anti-EMA, anti-CEA, and S100 protein is consistent with adnexal tumour with sweat gland differentiation. ${ }^{1}{ }^{2}$ 17-19

Perineural and intraneural invasion is a common and a particularly characteristic finding in SC (Fig 5). ${ }^{9}$ The SC extend deep into the dermis, often into subcutaneous fat, commonly into skeletal muscle (Fig 4), and sometimes into perichondrium, bone, vascular adventitia. $^{8-1020-22}$ The invasive tumour is locally destructive but regional lymph node or distant metastases have not been observed in well differentiated SC. Only one reported patient developed carcinoma in a lymph node, probably by direct perineural extension from the adjacent primary neoplasm. ${ }^{9}$ It is possible that our patient 2 has an SC of the eyelid with orbital involvement. In case 3, the tumour was very invasive and extended deep into the orbit.

A correct histological diagnosis is of importance because of the local aggressive nature of the tumour. Many difficulties are encountered in diagnosing this tumour histopathologically. ${ }^{15}$ When the architectural pattern is not present (shaved off or superficially punched out specimen), the characteristic histological features may not be apparent and the neoplasm can easily be misinterpreted as syringomas, trichoepitheliomas, or trichoadenomas, or squamous cell carcinoma. In case 2 , the biopsy performed contained superficial tissue only and, therefore, the deeper located SC was not present in the specimen. Later, a wider excision biopsy revealed an SC. If the architectural pattern is indicative of a malignant neoplasm but ductal differentiation is overlooked, an erroneous diagnosis of morphea-like basal cell or squamous cell carcinoma is made as occurred in two of our 
patients. Case 3 showed that various malignant skin and adnexal carcinomas may exist in the same patients (Fig 6). It is possible that these tumours were induced by the immunosuppressive agent cyclosporin. The eyelid/eyebrow tumour in this patient was a squamoid tumour without apparent eccrine differentiation, which has been diagnosed consecutively as squamous cell carcinoma, eccrine carcinoma with squamous cell carcinoma, and eventually as adnexal carcinoma with pilar differentiation. In the study of Leboit and Sexton, nine of 17 lesions were initially misdiagnosed because of the small size of the biopsy specimen. ${ }^{15}$

TREATMENT

Surgical excision is the oldest and probably the most reliable method of eradicating carcinomas of the eyelid. Because SCs tend to infiltrate far beyond the clinical margins of the neoplasm, surgical excision should be wider and deeper than anticipated and the excised specimens should be assessed carefully by the pathologist. Complete surgical excision under microscopical control must be performed.

Perineural invasion is frequently present in SC and may account for the high frequency of incomplete excision and local recurrence. In the literature, about $40-60 \%$ of patients had one or more local recurrences within 6 months to 30 years after treatment with standard wide local excision ${ }^{111223}$ Recurrent SC has to be treated by microscopically controlled surgery. ${ }^{24}$ When a SC infiltrates into the orbital fat or when perineural orbital involvement is detected, orbital exenteration has to be considered (see cases 2 and 3 ). In case 2, exenteration was advised but radiotherapy was eventually performed for different reasons, such as age and patient's preference. In the literature, radiation therapy is rarely applied and was used in three cases for the treatment of primary microcystic adnexal carcinoma. ${ }^{20} 25$ In two cases, results were documented and both had a recurrence within 2.5 to 3 years after radiotherapy. ${ }^{2025}$

\section{Conclusion}

SC is a rare, benign appearing but extremely invasive, locally destructive, slowly growing adnexal tumour. It may be mistaken clinically and microscopically for other benign and malignant entities. A delayed recognition, an incorrect initial histological interpretation of lesions, a standard surgical excision, and routine histopathological examination of excised tumour increase the risk of recurrence and morbidity or mortality. The aggressive tumour may result in mutilating procedures, such as orbital exenteration. Only tumour free margins of the excised tissue portend a favourable prognosis. ${ }^{9}$

The authors thank Dr Van Der Putte, pathologist of the department of pathology in the Academic Hospital Utrecht, for photographic assistance.

1 Abenoza P, Ackerman AB. Syringomatous carcinomas. In: Abenoza P, Ackerman AB, eds. Neoplasms with eccrine differentiation. Ackerman's histologic diagnosis of neoplastic skin disease: a method by pattern analysis. Philadelphia, London: Lea \& Febiger, 1990:373-412.

2 Goldstein DJ, Barr RJ, Cruz DJ. Microcystic adnexal carcinoma: a distinct clinicopathologic entity. Cancer 1982; 50:566-72

3 Lipper S, Peiper SC. Sweat gland carcinoma with syringomatous features: a light microscopic and ultrastrucsyringomatous features: a light micro
tural study. Cancer 1979;44:157-63.

4 Gulmen S, Pullon PA. Sweat gland carcinoma of the lips. Oral Surg 1976;41:643-9.

5 Kelly DE, Klein DM, Harrigan WF. Lip reconstruction following resection for an unusual basal cell carcinoma. Oral Surg 1975;40:19-26.

6 Requena L, Marquina A, Alegre V, Aliaga A, Sanchez Yus E. Sclerosing (microcystic adnexal) carcinoma: a tumour
from a single eccrine origin. Clin Exp Dermatol 1990;15: 222-4.

7 Santa Cruz DJ. Sweat gland carcinomas: a comprehensive review. Semin Diagn Pathol 1987;4:38-74.

8 Cooper PH, Mills SE. Microcystic adnexal carcinoma. $\mathcal{F}$ Am Acad Dermatol 1984;10:908-14.

9 Cooper PH, Mills SE, Leonard DD, Santa Cruz DJ, HeadCooper PH, Mills SE, Leonard DD, Santa Cruz DJ, Head-
ington JT, Barr RJ, et al. Sclerosing sweat duct (syringomaington JT, Barr RJ, et al. Sclerosing sweat duct (syringc

10 Glatt HJ, Proia AD, Tsoy EA, Fetter BF, Klintworth GK, Neuhaus R, et al. Malignant syringoma of the eyelid. Ophthalmology 1984;91:987-90.

11 Cooper PH. Sclerosing carcinomas of sweat ducts (microcystic adnexal carcinoma). Arch Dermatol 1986;122:261-4.

2 Sebastien TS, Nelson BR, Lowe L, Baker S, Johnson TM. Microcystic adnexal carcinoma. F Am Acad Dermatol 1993; 29:840-5.

13 Hesse RJ, Scharfenberg JC, Ratz JL, Griener E. Eyelid microcystic adnexal carcinoma. Arch Ophthalmol 1995;113: $494-6$.

14 Hunts JH, Patel BCK, Langer PD, Anderson, RL, Gerwels JW. Microcystic adnexal carcinoma of the eyebrow and eyelid. Arch Ophthalmol 1995;113:1332-3.

15 Leboit PE, Sexton M. Microcystic adnexal carcinoma of the skin. $\mathcal{F}$ Am Acad Dermatol 1993;29:609-18.

16 Keizer de RJW, Scheffer E. Masquerade of eyelid tumours. Doc Ophthalmol 1989;72:309-21.

17 Wick MR, Swanson PE. Cutaneous adnexal tumors. Chicago: ASCP Press, 1995:25-6.

18 Penneys NS, Nadji M, Ziegels-Weismann J, Ketabchi M, Morales AR. Carcinoembryonic antigen in sweat gland carcinomas. Cancer 1982;50:1608-11.

19 Penneys NS, Nadji M, Morales AR. Carcinoembronic antigen in benign sweat gland tumors. Arch Dermatol 1982;118:225-7.

20 Yuh WTC, Engelken JD, Whitaker DC, Dolan KD. Bone marrow invasion of microcystic adnexal carcinoma. Ann Otol Rhinol Laryngol 1991;100:601-3.

21 Birkby CS, Argenyi ZB, Whitaker DC. Microcystic adnexal carcinoma with mandibular invasion and bone marrow replacement. F Dermatol Surg Oncol 1989; 15:308-12.

22 Hamm JC, Argenta CL, Swanson NA. Microcystic adnexal carcinoma: an unpredictable aggressive neoplasm. Ann carcinoma: an unpredictable

23 Cooper PH. Carcinomas of sweat glands. In: Rosen PP, Fechne RE, eds. Pathol Annu 1987;22(pt1):83-124.

24 Burns MK, Chen SP, Goldberg LH. Microcystic adnexal carcinoma. Ten cases treated by Mohs micrographic surgery. F Dermatol Surg Oncol 1994;20:429-34.

25 Mayer MH, Winton GB, Smith AC, Lupton GP, Parry EL, Shagets FW. Microcystic adnexal carcinoma (sclerosing sweat duct carcinoma). Plast Reconstr Surg 1989;84:970-5. 